A LETTERS JOURNAL EXPLORING THE FRONTIERS OF PHYSICS

\title{
OFFPRINT
}

\section{Compressing nearly hard sphere fluids increases glass fragility}

\author{
L. Berthier and T. A. Witten \\ EPL, 86 (2009) 10001
}

Please visit the new website

www.epljournal.org 


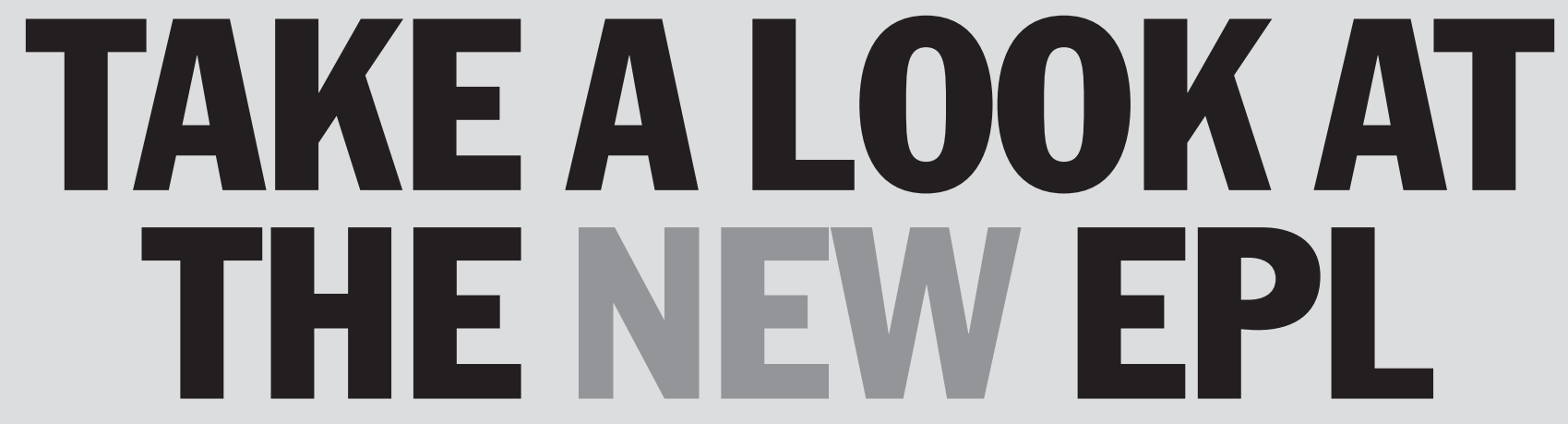

\section{Europhysics Letters (EPL) has a new online home at www.epljournal.org}

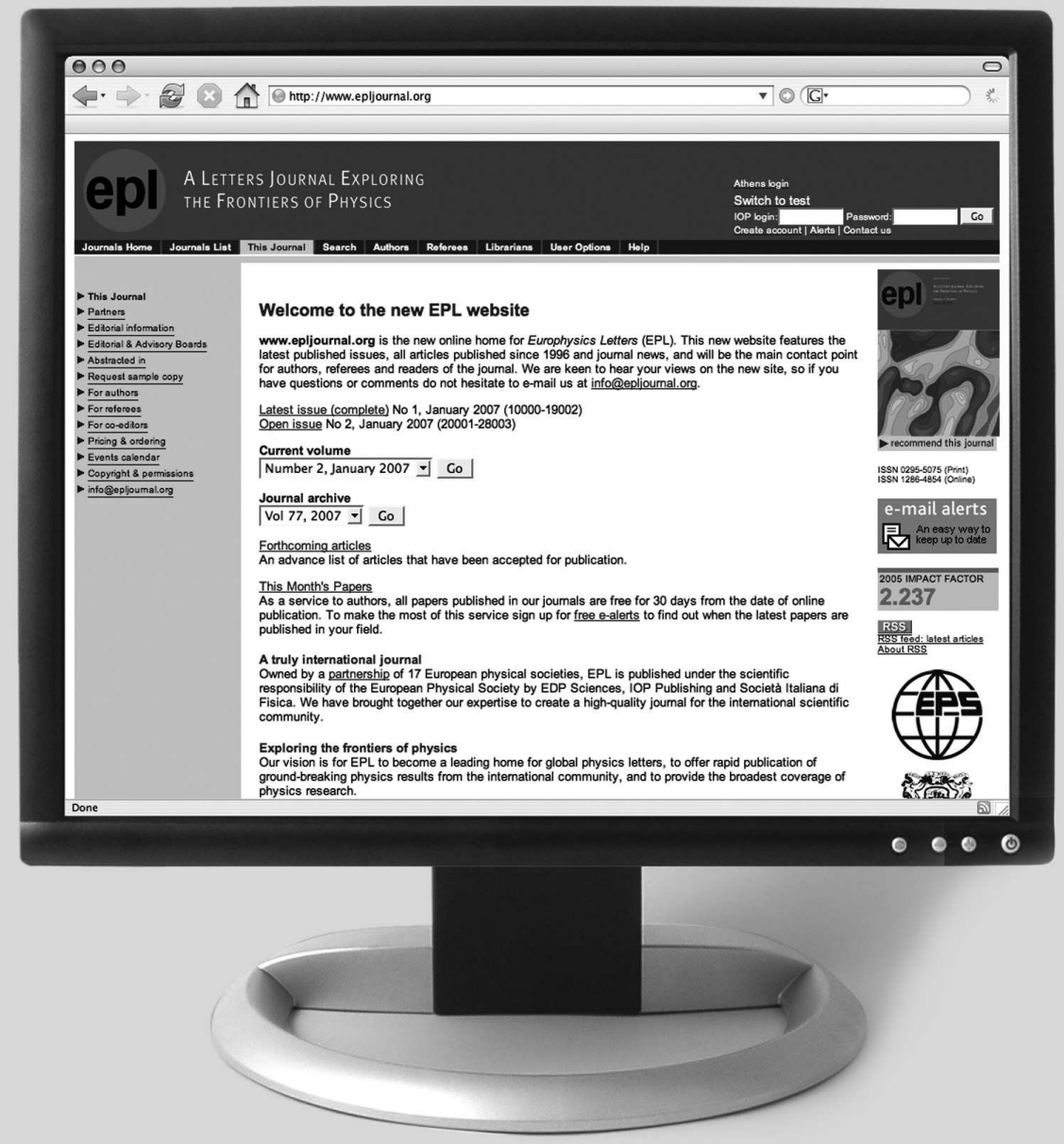

Take a look for the latest journal news and information on:

- reading the latest articles, free!

- receiving free e-mail alerts

- submitting your work to EPL 


\title{
Compressing nearly hard sphere fluids increases glass fragility
}

\author{
L. Berthier ${ }^{1(a)}$ and T. A. Witten ${ }^{2}$ \\ ${ }^{1}$ Laboratoire des Collö̈des, Verres et Nanomatériaux, UMR 558\%, Université Montpellier II and CNRS \\ 34095 Montpellier, France, EU \\ 2 James Franck Institute and Department of Physics, The University of Chicago - 929 E. 57th Street, \\ Chicago, IL 6063\%, USA
}

received on 23 February 2009; accepted by L. F. Cugliandolo on 11 March 2009 published online 8 April 2009

PACS 05.10.-a - Computational methods in statistical physics and nonlinear dynamics

PACS 05.20.Jj - Statistical mechanics of classical fluids

PACS 64.70.P- - Glass transitions of specific systems

\begin{abstract}
We use molecular dynamics to investigate the glass transition occurring at large volume fraction, $\varphi$, and low temperature, $T$, in assemblies of soft repulsive particles. We find that equilibrium dynamics in the $(\varphi, T)$-plane obey a form of dynamic scaling in the proximity of a critical point at $T=0$ and $\varphi=\varphi_{0}$, which should correspond to the ideal glass transition of hard spheres. This glass point, "point $G$ ", is distinct from athermal jamming thresholds. A remarkable consequence of scaling behaviour is that the dynamics at fixed $\varphi$ passes smoothly from that of a strong glass to that of a very fragile glass as $\varphi$ increases beyond $\varphi_{0}$. Correlations between fragility and various physical properties are explored.
\end{abstract}

Copyright (C) EPLA, 2009

Introduction. - Structureless, hard, frictionless particles pass from a mobile to an immobile state with increasing density [1]. Interacting particles and molecules in a glass-forming material also pass from a mobile fluid state to an immobile glassy state as the temperature is reduced [2]. Much research in the last decade has been devoted to extracting a common geometric essence from these two classes of phenomena. One line of research approaches the threshold of immobilization or jamming via processes unrelated to thermal equilibrium [3-6]. Connections between these jamming transitions and those seen at positive temperature are suggested [7], but remain unclear. In particular, the threshold density for jamming and that for immobilization at non-zero temperature are considered identical by some researchers $[8,9]$, distinct for others [10-12], ill-defined by some others [3,13]. Direct measurements are not conclusive, because the location of the glass transition relies upon fitting and extrapolation $[8,10]$, while the jamming transition is not uniquely defined $[3,4]$.

The notion that temperature and density should have analogous effects on the glass transition has a long history [14,15], although quantitative evidence supporting these analogies is limited. Recent work studying the effect of pressure on the glass transition showed that the

\footnotetext{
(a) E-mail: ludovic.berthier@lcvn.univ-montp2.fr
}

dynamics of glass-formers is little affected by increasing the density, since a simple rescaling procedure collapses a broad range of dynamic data $[16,17]$. This finding directly implies that the (isochoric) fragility [18] of most glass-formers is independent of density, at least in the range currently explored by experiments. A second conclusion is that the glass transition of molecular systems is mostly controlled by temperature, suggesting that the density-driven glass transition of hard spheres might have a different nature.

In this paper, we study the relative influence of density and temperature on the glass transition using a model of soft repulsive particles [19]. In the zero-temperature limit, the model is equivalent to density-controlled hard spheres, while it resembles thermally driven dense fluids at large density and finite temperature. Another motivation to use compressible particles is to access densities beyond the hard sphere critical density for kinetic arrest which are unreachable with the hard sphere potential. This approach has proven useful in the context of athermal jamming [4-6], but it was not extended to thermal equilibrium before. At equilibrium, issues related to the possible protocol dependence of the results [20] do not arise.

Using computer simulations we have studied the equilibrium dynamics of a three-dimensional assembly of soft repulsive particles varying the volume fraction, $\varphi$, and temperature, $T$ (see fig. 1). We have discovered a simple 


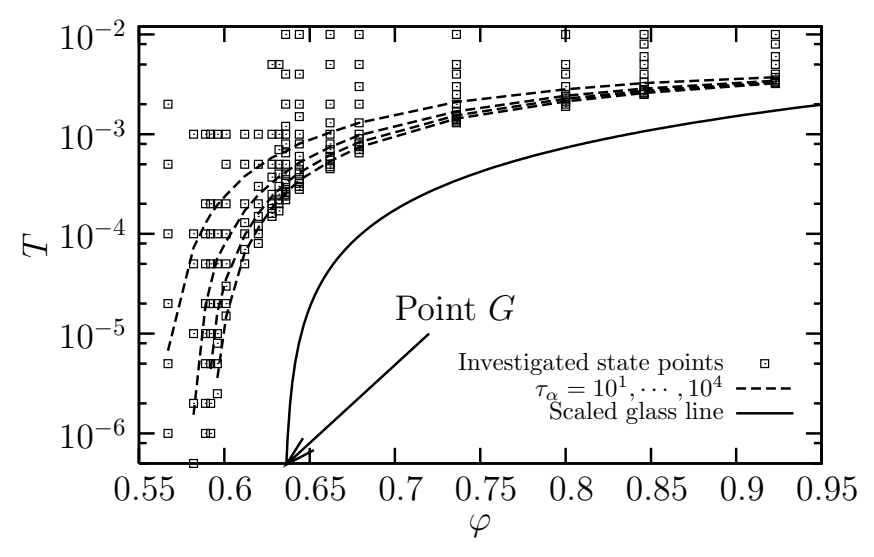

Fig. 1: Volume fraction, $\varphi$, and temperature, $T$, phase diagram of elastic spheres with investigated state points. Four isorelaxation time (dashed) lines are displayed, showing that $\tau_{\alpha}$ increases by increasing $\varphi$ or decreasing $T$. Scaling permits accurate determination of the glass point $G$ at $T=0$ and $\varphi_{0}=0.635$. The glass transition (full) line is obtained assuming a specific form of the scaling function in eq. (4).

connection between density and temperature effects, from which several interesting results are deduced. Equilibrium dynamics obey critical scaling in the proximity of a glass critical point, which we call "point $G$ ", at $T=0$ and $\varphi=\varphi_{0}$, see fig. 1. By approaching point $G$ along various equilibrium routes, we can determine the functional form of the dynamics and the location of the singularity with much less uncertainty than is usually possible in glasses. In particular, we confirm the non-trivial density dependence of the relaxation time suggested by recent experiments on colloidal hard spheres [10]. Scaling also implies that the evolution of the dynamics with temperature at fixed $\varphi$ passes smoothly from that of a strong glass to that of a very fragile glass as $\varphi$ increases beyond $\varphi_{0}$. Compared to previous numerical glass models with tunable fragility, the present model does not require changing the composition of the liquid [21], or the curvature of space [22], fragility varies over a much broader range [23], and provides a new conceptual way to tune fragility.

Model and methods. - We use molecular dynamics simulations [24] to study a system composed of $N$ particles enclosed in a periodic cube of linear size $L$ and interacting through a pair-wise potential: $V\left(r_{i j}\right)=\epsilon\left(1-r_{i j} / \sigma_{i j}\right)^{2}$ for $r_{i j}<\sigma_{i j}, V\left(r_{i j}\right)=0$ otherwise. The interparticle distance is $r_{i j}=\left|\mathbf{r}_{i}-\mathbf{r}_{j}\right|$ and $\sigma_{i j}=\left(\sigma_{i}+\sigma_{j}\right) / 2$, where $\mathbf{r}_{i}$ and $\sigma_{i}$ are the position and diameter of particle $i$, respectively. We use system sizes between 500 and 8000 particles, and report results for $N=1000$, for which no finite size effects are detected, within numerical accuracy. We prevent crystallization by using a 50:50 binary mixture of spheres of diameter ratio 1.4 [4]. The volume fraction is $\varphi=\frac{\pi N}{12 L^{3}}\left(1+1.4^{3}\right) \approx 0.98 \rho$, with $L$ expressed in units of the small particle diameter and $\rho=N / L$ the number density. Up to volume fraction $\varphi=0.846$ we detect no sign of crystallization at all studied temperatures; above $\varphi=0.924$ there was evidence of incipient crystallization at the lowest temperatures. However, these crystallization effects occur well away from the region of interest around $\varphi_{0}=0.635$. We use $\epsilon$ as the energy unit, and $\sqrt{\sigma_{2}^{2} / \epsilon}$ as time unit, masses are set to unity. All dynamical results are obtained at thermal equilibrium, which has been carefully controlled. When temperature is low and density is large, we are not able to thermalize. Crystallization and equilibrium issues determine the boundaries of the region investigated in the phase diagram of fig. 1.

Dynamic scaling at thermal equilibrium. - In fig. 2(a), we report the evolution of the averaged relaxation timescale, $\tau_{\alpha}(\varphi, T)$ for all investigated state points. We quantify the microscopic dynamics through the self-part of the intermediate scattering function

$$
F_{s}(q, t)=\frac{1}{N_{b}}\left\langle\sum_{j=1}^{N_{b}} \mathrm{e}^{i \mathbf{q} \cdot\left(\mathbf{r}_{j}(t)-\mathbf{r}_{j}(0)\right)}\right\rangle,
$$

where $\mathbf{q}$ is the scattering vector $(q=6.1$, close to the first diffraction peak) and angular brackets indicate a thermal average. We define $\tau_{\alpha}$ by $F_{s}\left(q, \tau_{\alpha}\right)=\mathrm{e}^{-1}$, and we arbitrarily choose to restrict the average in (1) to the $N_{\mathrm{b}}$ big particles. Data for $\tau_{\alpha}$ are normalized by $1 / \sqrt{T}$, which is equivalent to renormalizing times by the "thermal" time $\frac{1}{v_{\mathrm{MB}}}$, where $v_{\mathrm{MB}}$ is the first moment of the MaxwellBoltzmann distribution. Therefore, in the $T \rightarrow 0$ limit where particle overlaps are energetically disfavoured, the dynamics of the elastic spheres coincide with that of hard spheres thermalized at $T=1$. We have verified this equivalence quantitatively by comparing our numerical results to those of the hard sphere studies presented in ref. [10].

The temperature evolution of $\tau_{\alpha}$ exhibits two qualitatively distinct regimes. For dilute systems, $\tau_{\alpha}$ first increases when $T$ decreases, but saturates when $T \rightarrow 0$ at a finite value corresponding to $\tau_{\alpha}^{\mathrm{hs}}(\varphi)$, the relaxation time of the hard sphere fluid. For dense systems, $\tau_{\alpha}$ seems to increase with no saturation, and we cannot reach equilibrium when $T$ becomes too small and the system becomes an unequilibrated glass (see fig. 1). The frontier between these two regimes must correspond to $\varphi=\varphi_{0}$, the density at which $\tau_{\alpha}^{\mathrm{hs}}(\varphi)$ diverges. By analogy with the Jamming transition occurring at point $J$ along the $\varphi$-axis for athermal systems of spheres [4], we call "point $G$ " the location of the Glass transition at $\varphi_{0}$ along the $\varphi$-axis for thermal systems.

We now formulate the hypothesis that dynamics in the proximity of point $G$ obey scaling behaviour. We surmise that elastic spheres at $T>0$ behave, in a statistical sense, similarly to hard spheres with a reduced diameter [14]. Physically, we assume that at low $T$, overlaps between particles are very small, and so are the corrections to hard sphere behaviour. Below, we justify why an accurate relation between density and temperature is

$$
\varphi_{\mathrm{eff}}(\varphi, T) \approx \varphi-a T^{\mu / 2},
$$



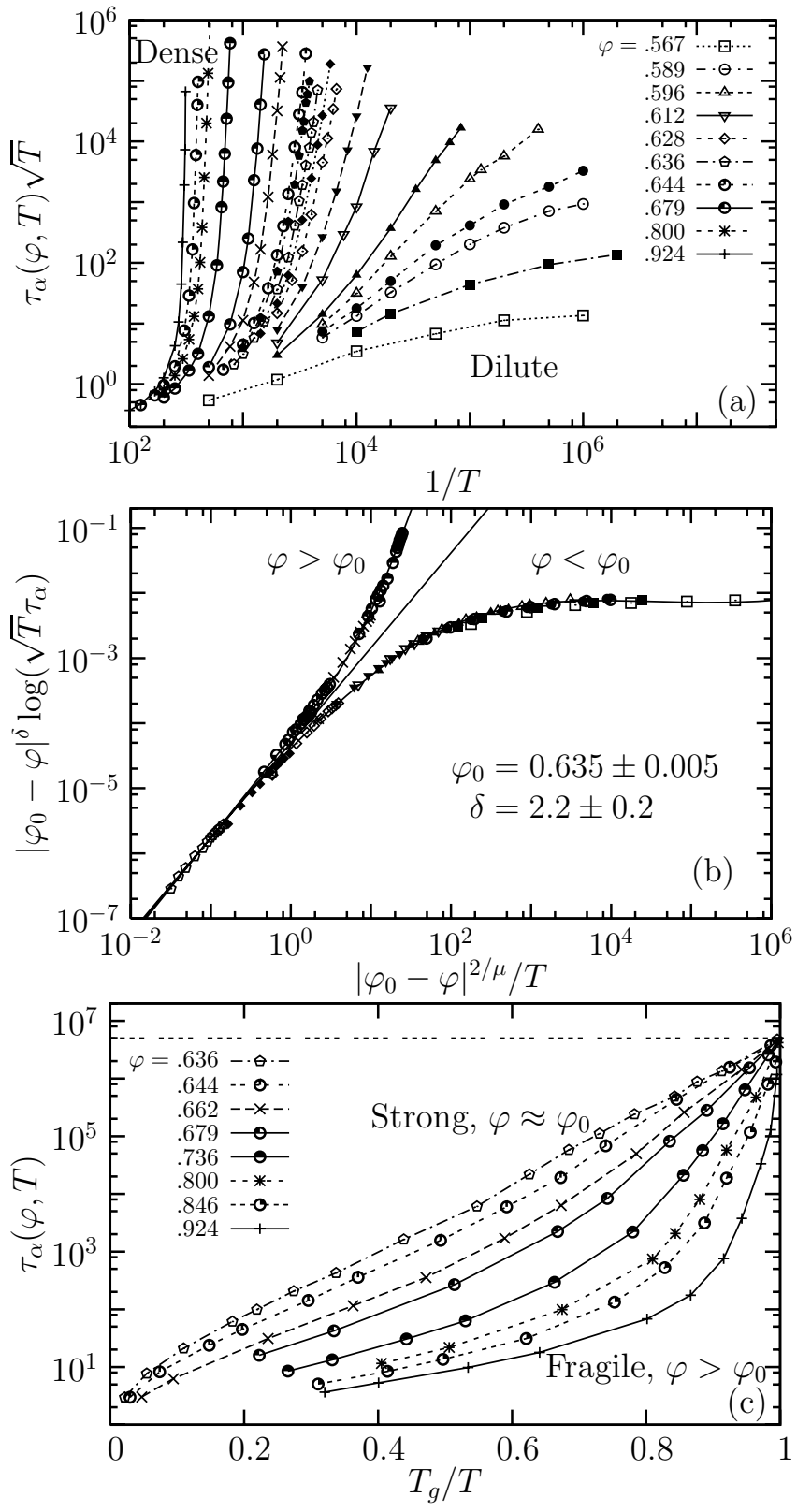

Fig. 2: (a) Relaxation timescales for all investigated state points. We rescale $\tau_{\alpha}$ by $1 / \sqrt{T}$ so that the $T \rightarrow 0$ limit coincides with hard spheres thermalized at $T=1$. Not all volume fractions are labelled. (b) Collapse of the $\varphi<\varphi_{0}$ and $\varphi>\varphi_{0}$ data along the bottom and top branches, respectively, using eq. (4) and imposing $\mu=1.3$ from considering the potential energy. (c) Arrhenius plot of the $\varphi>\varphi_{0}$ data, using the definition $\tau_{\alpha}\left(\varphi, T_{g}\right)=5 \cdot 10^{6}$.

where $a>0$ is a numerical prefactor, $\mu>0$, a characteristic exponent and $\varphi_{\text {eff }}(\varphi, T)<\varphi$, an effective volume fraction for the elastic sphere system at volume fraction $\varphi$ and temperature $T$. Furthermore, we build upon a recent analysis of the dynamics of colloidal hard spheres [10] and assume an exponential divergence for $\tau_{\alpha}^{\text {hs }}$ :

$$
\tau_{\alpha}^{\mathrm{hs}}(\varphi) \sim \exp \left[\frac{A}{\left(\varphi_{0}-\varphi\right)^{\delta}}\right]
$$

where $\delta \approx 2$ and $\varphi_{0} \approx 0.637$ [10]. Although the exponential divergence of $\tau_{\alpha}^{\mathrm{hs}}$ is unambiguous from hard sphere studies [10], the values of $\delta$ and $\varphi_{0}$ remain subject to large uncertainty because the divergence must be extrapolated along a single path (increasing $\varphi$ at $T=0$ ), relatively far away from $\varphi_{0}$.

We analyze the results of fig. 2(a) and approach point $G$ from multiple paths in the $(\varphi, T)$-plane to establish the robustness of eq. (3). Combining (2) and (3) we suggest

$$
\tau_{\alpha}(\varphi, T) \sim \exp \left[\frac{A}{\left|\varphi_{0}-\varphi\right|^{\delta}} F_{ \pm}\left(\frac{\left|\varphi_{0}-\varphi\right|^{2 / \mu}}{T}\right)\right]
$$

where the scaling functions $F_{ \pm}(x)$ apply to densities above/below $\varphi_{0}$. We expect therefore that $F_{-}(x \rightarrow \infty) \rightarrow 1$ to recover the hard sphere fluid limit, eq. (3), when $T \rightarrow 0$ and $\varphi<\varphi_{0}$. Similarly, $F_{+}(x \rightarrow \infty) \rightarrow \infty$. Moreover, continuity of $\tau_{\alpha}$ at finite $T$ and $\varphi=\varphi_{0}$ implies $F_{-}(x \rightarrow 0) \sim$ $F_{+}(x \rightarrow 0) \sim x^{\delta \mu / 2}$. Dynamic scaling was recently observed for athermal jamming transitions $[5,6]$, but the nature of the critical density and hard sphere divergence (algebraic instead of exponential) were different from eq. (4), while no physical interpretation of scaling in terms of an effective hard sphere behaviour was offered.

The proposed scaling behaviour is confirmed in fig. 2(b) for data in the range $\varphi \in[0.567,0.736]$. To obtain the scaling plot, we fix $\mu=1.3$ (see below), and use $\varphi_{0}$ and $\delta$ as free parameters to collapse $\left|\varphi_{0}-\varphi\right|^{\delta} \log \tau_{\alpha}$ against $\left|\varphi_{0}-\varphi\right|^{2 / \mu} / T$. The best collapse is shown, but good results are obtained for a range of values of $\varphi_{0}$ and $\delta$, yielding error bars

$$
\varphi_{0}=0.635 \pm 0.005, \quad \delta=2.2 \pm 0.2
$$

Outside this range, the collapse quickly deteriorates. Note that $\delta=1$, often used to describe hard sphere data [8], is inconsistent with our results. Our scaling analysis thus lends crucial support to the conclusions of [10]. Of course, we cannot exclude that a different dynamic regime can be entered when relaxation timescales beyond the reach of our numerical capabilities are added to the analysis.

Glass fragility. - The scaling in eq. (4) predicts the temperature dependence of $\tau_{\alpha}$ at $\varphi_{0}$ : $\tau_{\alpha}\left(\varphi_{0}, T\right) \sim \exp \left(A / T^{\mu \delta / 2}\right)$. Since $\mu \delta / 2 \approx 1.43$, this divergence is slightly stronger than, but not very different from, the simple Arrhenius behaviour observed for "strong" glass-formers [18]. The divergence of the scaling function $F_{+}(x)$ for large argument moreover implies that the temperature dependence of $\tau_{\alpha}$ for $\varphi>\varphi_{0}$ becomes steeper, making the materials increasingly "fragile" [18]. This is vividly demonstrated in fig. 2(c), where we conventionally rescale $T$ by $T_{g}$ defined as the temperature where $\tau_{\alpha}$ reaches an arbitrary value [18], $\log _{10} \tau_{\alpha}\left(\varphi, T_{g}\right)=X_{g}$. Such a large change of fragility was not reported in a particle model before [21-23]. Here, it directly results from the interplay between $\varphi$ and $T$. We quantify fragility 

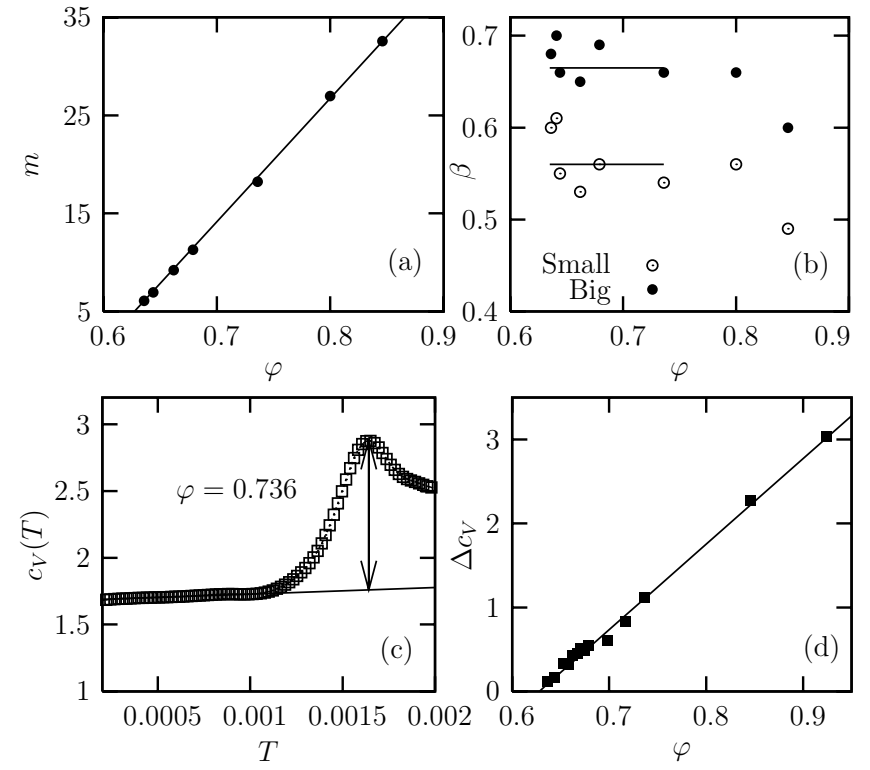

Fig. 3: (a) $\varphi$ dependence of the steepness index, eq. (6), measured from the data in fig. 2(c). The line is the linear fit predicted in eq. (7). (b) The stretching exponent describing the time decay of $F_{s}(q, t)$ for both species coincides with the one for hard spheres in the scaling regime of fig. 2(b), uncorrelated to fragility. (c) Temperature dependence of the specific heat at $\varphi=0.736$ obtained by heating the glass slowly. The full line fits the $T$-dependence of $c_{V}(T)$ below $T_{g}$, allowing the definition of $\Delta c_{V}$, as shown by the vertical arrow. (d) The $\varphi$-dependence of $\Delta c_{V}$ (symbols) is well described by a linear increase (full line), closely tracking the fragility in (a).

by the steepness index [18]

$$
m=\left.\frac{\partial \log _{10} \tau_{\alpha}}{\partial\left(T_{g} / T\right)}\right|_{T_{g}},
$$

which increases steadily when $\varphi$ increases beyond $\varphi_{0}$, see fig. 3(a). The linear variation can be rationalized realizing that an approximate expression for $F_{+}(x)$ in eq. (4) can be obtained by pushing further our correspondence between elastic and hard spheres, and assuming: $\tau_{\alpha}(\varphi, T) \approx$ $\tau_{\alpha}^{\mathrm{hs}}\left[\varphi_{\mathrm{eff}}(\varphi, T)\right]$. This gives $F_{+}(x) \approx\left(a x^{-\mu / 2}-1\right)^{-\delta}$, from which we get

$$
m\left(\varphi_{0}+\Delta \varphi\right) \approx m_{0}(1+\alpha \Delta \varphi)
$$

where $m_{0}=\mu \delta X_{g} / 2$ and $\alpha=a\left(X_{g} / A\right)^{1 / \delta}$. Figure 3(a) shows that this predicted linear behaviour is accurately obeyed over a seven-fold range of fragility $m$. A broader range of $m$ would be obtained for $X_{g}$ corresponding to the laboratory $T_{g}$. Multiplying, for instance, $X_{g}$ by a factor 3 (from $X_{g}=5$ to 15 ) in eq. (7) yields $m \in[18,150]$, quite close to the experimental spectrum for the fragility of glass-formers [18].

Additionally, the explicit, but approximate, expression for $F_{+}(x)$ predicts the location of a glass line,
$T_{0}(\varphi)=\left[\left(\varphi-\varphi_{0}\right) / a^{1 / \delta}\right]^{2 / \mu}$, shown in fig. 1 , in the spirit of [15]. Unfortunately, our numerical data alone do not allow us to determine whether $F_{+}(x)$ indeed diverges for a finite value of its argument. Therefore, while the location of point $G$ is very much constrained by our data, the existence of a finite temperature singularity for $\varphi>\varphi_{0}$ remains open.

Different glasses possess different fragilities, but this variability is not satisfactorily understood. Instead, experimentalists often correlate the properties of a glass to its fragility [18]. Having a model with tunable fragility allows theoretical exploration of the part of these correlations that is not due to the variety of structures (e.g., networks and polymer chains) observed in real glass-formers. We have studied two popular correlations: the stretching of time correlation functions and the specific heat jump at $T_{g}$. We fit the alpha-relaxation in $F_{s}(q, t)$ for both species with a stretched exponential form: $F_{s} \sim \mathrm{e}^{-(t / \tau)^{\beta}}$. We find that $\beta$ is very weakly $T$-dependent, and report its value for different $\varphi$ in fig. 3(b). For $\varphi$ within the scaling regime of fig. $2(\mathrm{~b})$, the observed $\beta$ 's for small and big particles are independent of compression, and hence of fragility, but are consistent with those of hard spheres observed previously [10]. While some degree of correlation is often reported in experiments [2], it is not very strong when data for polymers are discarded from the analysis [25], which is in agreement with our findings.

We measure the potential energy, $V_{\text {pot }}(\varphi, T)=$ $\left\langle\sum_{i<j} V\left(r_{i j}\right)\right\rangle / N$, and the specific heat, $c_{V}=\mathrm{d} V_{\text {pot }} / \mathrm{d} T$. Mimicking experiments, we obtain the jump in $c_{V}$ at the glass transition by slowly cooling the system, at constant $\varphi$, down to $T=0$ before re-heating at the same rate. For all $\varphi$, we use very slow rates, $\frac{1}{T_{g}} \frac{\mathrm{d} T}{\mathrm{~d} t} \approx 3 \cdot 10^{-7}$. The typical behaviour of $c_{V}(T)$ upon heating is shown in fig. 3(c). As in experiments, a peak is observed when equilibrium is recovered. We estimate $\Delta c_{V}$ as the difference between the peak height and the glass specific heat, although different estimates yield qualitatively similar results. The $\varphi$-dependence of $\Delta c_{V}$ is shown in fig. 3(d), together with a linear fit. As suggested by experiments [2,18], we find a linear relation between fragility and specific heat.

To qualitatively explain this observation we discuss the behaviour of $V_{\text {pot }}$. For a very dilute system, $V_{\text {pot }}$ decreases rapidly as $T$ decreases. A collision-based analysis shows that $V_{\text {pot }} \sim T^{3 / 2}$ : the energy decreases faster than linearly and vanishes at $T=0$. Correspondingly, the "excess" energy above the hard sphere ground state $\left(V_{\text {pot }}=0\right)$ is small, explaining the smallness of $\Delta c_{V}$ at small volume fraction. For very dense systems, the energy decreases linearly at low $T$, and increases with $\varphi$ since particles overlap more upon compression. Therefore the larger $\varphi$, the larger $c_{V}$ in the fluid phase, and the larger is $\Delta c_{V}$, as observed in fig. 3(d). These considerations suggest that further analysis of the potential enery landscape properties of the present system and comparison with model landscapes $[26,27]$ could be very valuable. 
In a broad density range encompassing $\varphi_{0}$, simulations suggest $V_{\text {pot }} \sim T^{\mu}$, with $\mu \approx 1.3$, a value intermediate between the dilute and dense limits. Due to the harmonic nature of the potential, $\sqrt{V_{\text {pot }}}$ represents the average overlap between interacting particles, and suggests a way to quantitatively estimate $\varphi_{\text {eff }}$ in eq. (2), and to justify the form of the scaling variable in eq. (4). These energetic considerations are a suggestive physical interpretation, rather than a rigorous derivation, of the success of eq. (2) at collapsing data in fig. 2(c).

Non-equilibrium jamming at $T=0$. - Finally, the low- $T$, non-equilibrium behaviour of the energy density during slow annealing is also informative. For $\varphi<\varphi^{\star} \approx 0.662, V_{\text {pot }}(\varphi, T \rightarrow 0)=0$, while $V_{\text {pot }}$ remains finite above $\varphi^{\star}$. Therefore, the nature of the $T=0$ glasses produced by slow annealing at volume fractions above point $G$ changes at $\varphi^{\star}$ where a "jamming" transition similar to the one described in [4] occurs. Note that $\varphi^{\star}$ is larger than $\varphi_{J} \approx 0.648$, the critical density for jamming determined in [4] for the same system, because our glasses have been annealed, with no trace of demixing or crystallization $[3,13]$. We emphasize that these jamming transitions occur at different densities when different protocols are used [13], while the equilibrium glass transition at $\varphi_{0}$ analyzed in this work is uniquely defined by the behaviour of the equilibrium relaxation time, so that both transitions should be considered as distinct phenomena [12].

Discussion and conclusion. - The dynamic scaling behaviour in eq. (4) describing the interplay between density and temperature for soft repulsive particles is in stark contrast with the results obtained for soft spheres interacting with a pure inverse power law potential $[16,17]$, because the interaction potential of elastic spheres has a finite cutoff (the particle diameter). Therefore, elastic spheres reduce to hard spheres in the small- $T$ limit, which is crucial to obtain a change in fragility. We believe our results would generically carry out for repulsive potentials with a finite range.

A second interesting feature of this study is that the location of a divergence for the equilibrium relaxation time is extrapolated from a demanding scaling procedure where a large set of independent data is used to locate a critical density. To the best of our knowledge, such an analysis using two control parameters has no counterpart in the glass transition literature. In particular, we believe it yields a rather accurate determination of the critical density $\varphi_{0}$ where the equilibrium relaxation time of the hard sphere fluid extrapolates to $\infty$, but leaves open the existence of a finite temperature singularity above $\varphi_{0}$. Our determination of the existence of a genuine divergence of $\tau_{\alpha}$ for hard spheres at $\varphi_{0}$ is based on the analysis of the metastable fluid, demixing and crystallization being avoided due to polydispersity. Whether this ideal glass transition is itself avoided [13] due to crystallization or demixing at much larger density is another important issue that our data leave open.
In conclusion, our preliminary studies of repulsive elastic particles at thermal equilibrium suggest that soft repulsive particles are a promising new tool to gain deeper understanding of glass transition phenomena in colloidal and molecular systems. Experimentally, this could be directly realized using soft colloidal particles.

Fruitful exchanges with G. Biroli, P. Chaudhuri, L. Cipelletti, W. Kob, J. Kurchan, S. Nagel, D. Reichman, and G. TARJus are acknowledged. LB thanks the University of Chicago and Argonne National Laboratory for the financial support in the early stages of this work in 2007.

\section{REFERENCES}

[1] Jaeger H. M., Nagel S. R. and Behringer R. P., Rev. Mod. Phys., 68 (1996) 1259.

[2] Debenedetti P. G. and Stillinger F. H., Nature, 410 (2001) 259.

[3] Torquato S., Truskett T. M. and Debenedetti P. G., Phys. Rev. Lett., 84 (2000) 2064.

[4] O'Hern C. S., Langer S. A., Liu A. J. and Nagel S. R., Phys. Rev. Lett., 88 (2002) 075507.

[5] Olsson P. and Teitel S., Phys. Rev. Lett., 99 (2007) 178001.

[6] Hatano T., J. Phys. Soc. Jpn., 77 (2008) 123002.

[7] Liu A. J. and Nagel S. R., Nature, 396 (1998) 21.

[8] Cheng Z., Zhu J., Chaikin P. M., Phan S. and Russel W. B., Phys. Rev. E, 65 (2002) 041405.

[9] Schweizer K. S., J. Chem. Phys., 127 (2007) 164506.

[10] Brambilla G., El Masri D., Pierno M., Petekidis G., Schofield A. B., Berthier L. and Cipelletti L., Phys. Rev. Lett., 102 (2009) 085703.

[11] Parisi G. and Zamponi F., J. Chem. Phys., 123 (2005) 144501.

[12] Krzakala F. and Kurchan J., Phys. Rev. E, 76 (2007) 021122 .

[13] Donev A., Stillinger F. H. and Torquato S., J. Chem. Phys., 127 (2007) 124509.

[14] Cohen M. H. and Turnbull D., J. Chem. Phys., 31 (1959) 1164.

[15] Hudson S. and Andersen H. C., J. Chem. Phys., 69 (1978) 2323.

[16] Roland C. M., Hensel-Bielowka S., Paluch M. and Casalini R., Rep. Prog. Phys., 68 (2005) 1405.

[17] Tarjus G., Kivelson D., Mossa S. and AlbaSimionesco C., J. Chem. Phys., 120 (2004) 6135.

[18] Angell C. A., Science, 267 (1995) 1924.

[19] Durian D. J., Phys. Rev. Lett., 75 (1995) 4780.

[20] Donev A., Torquato S., Stillinger F. H. and Connelly R., Phys. Rev. E, 70 (2004) 043301.

[21] Coslovich D. and Pastore G., J. Chem. Phys., 127 (2007) 124504.

[22] Sausset F., Taruus G. and Viot P., Phys. Rev. Lett., $101(2008) 155701$. 
[23] Sastry S., Nature, 409 (2000) 164.

[24] Allen M. and Tildesley D., Computer Simulation of Liquids (Oxford University Press, Oxford) 1987.

[25] Heuer A., J. Phys.: Condens. Matter, 20 (2008) 373101.
[26] Heuer A. and Saksaengwijit A., Phys. Rev. E, 77 (2008) 061507.

[27] Moreno A. J., Saika-Voivod I., Zaccarelli E., La Nave E., Buldyrev S. V., Tartaglia P. and Sciortino F., J. Chem. Phys., 124 (2006) 204509. 